UTHEP-453

UTCCP-P-119

November, 2001

\title{
Elementary Particles on a Dedicated Parallel Computer
}

\author{
Kazuyuki Kanaya ${ }^{1}$ for the CP-PACS Collaboration? \\ ${ }^{1}$ Institute of Physics, University of Tsukuba, Tsukuba, Ibaraki 305-8571, Japan \\ kanaya@rccp.tsukuba.ac.jp
}

\begin{abstract}
Numerical simulation of lattice QCD is now an indispensable tool for investigating non-perturbative properties of quarks at low energies. In this paper, I introduce a recent lattice QCD calculation of light hadron spectrum and quark masses performed on a dedicated parallel computer, CP-PACS. From a simulation of QCD with two flavors of dynamical quarks, we find $m_{u d}^{\overline{\mathrm{MS}}}(2 \mathrm{GeV})=3.44_{-0.22}^{+0.14} \mathrm{MeV}$ for the averaged mass of $u$ and $d$ quarks to reproduce the experimental masses of $\pi$ and $\rho$ mesons, and $m_{s}^{\overline{\mathrm{MS}}}(2 \mathrm{GeV})=88_{-6}^{+4}$ $\mathrm{MeV}$ or $90_{-11}^{+5} \mathrm{MeV}$ to reproduce the $K$ or $\phi$ meson mass. These values are about $20-30 \%$ smaller than the previous estimates using the quenched approximation.
\end{abstract}

\section{Introduction}

Quarks are among the most fundamental building blocks of nature. Unlike the previous elementary constituents, quarks are found to possess a remarkable property, confinement, i.e., we cannot isolate quarks by dissociating hadrons. Historically, fundamental theories of nature have emerged through reactions of isolated elements to weak external perturbations. Quark confinement, however, has the consequence that we cannot directly measure the fundamental properties of quarks by experiment.

Fortunately, quarks are found to possess another remarkable property, asymptotic freedom, whereby interactions among quarks become weak at high energies. This enabled us to identify quantum chromodynamics (QCD) as the fundamental theory of quarks. QCD has been quite successful in explaining high energy processes of particles.

On the other hand, because the coupling parameter of QCD becomes large at low energies, basic properties of hadrons, such as mass spectrum, spatial sizes, decay constants, etc., are not calculable by conventional analytic methods. In other words, we cannot reconstruct hadrons from quarks based on the dynamics of QCD yet. In order to answer

${ }^{2}$ CP-PACS Collaboration: A. Ali Khan, S. Aoki, G. Boyd, R. Burkhalter, S. Ejiri, M. Fukugita, S. Hashimoto, K. Ide, K.-I. Ishikawa, N. Ishizuka, Y. Iwasaki, T. Izubuchi, K. Kanaya, T. Kaneko, Y. Kuramashi, V. Lesk, T. Manke, Y. Namekawa, K.-I. Nagai, J.-I. Noaki, M. Okawa, M. Okamoto, Y. Taniguchi, H.P. Shanahan, A. Ukawa, T. Umeda, T. Yamazaki, and T. Yoshié

Talk presented at the 18th AvH symposium "100 Years Werner Heisenberg - Works and Impact", Bamberg, Germany, September 26-30, 2001. 
the fundamental question of whether QCD is correct also at low energies, we have to carry out non-perturbative calculations of hadrons directly from the first principles of QCD.

Calculation of low-energy hadron properties is also required for determination of fundamental parameters of nature. Due to quark confinement, quark masses and coupling parameters have to be inferred indirectly from a comparison of experimental results for hadron masses etc. with a theoretical calculation of them as functions of the fundamental parameters of QCD. Also for electroweak interactions, although the core part of the reaction can be reliably calculated in perturbation theory, uncertainties in QCD corrections to the quark currents propagate to the determination of the Cabibbo-Kobayashi-Maskawa parameters.

Numerical simulation of quarks based on the lattice formulation of QCD is currently the only method to calculate low-energy non-perturbative properties of hadrons. Recently, predictions with big impact are beginning to be produced through development of dedicated parallel computers [1]. In particular, a big breakthrough was achieved by the CP-PACS computer developed at the University of Tsukuba [2]. In this paper, I summarize major results from CP-PACS, focusing on the most fundamental topics of QCD, i.e., the light hadron spectrum and light quark masses.

In Sec. 2, I introduce the lattice formulation of QCD and explain why dedicated computers have been developed by physicists. Results for the light hadron spectrum and light quark mass are presented in Secs. 3 and 4 . Conclusions are given in Sec. 5 .

\section{Lattice QCD and dedicated parallel computers}

We formulate QCD on a four-dimensional hypercubic lattice with a finite lattice spacing $a$ and finite lattice size $N a$. The real world is defined by the limit of vanishing lattice spacing $a \rightarrow 0$ keeping the lattice size $N a$ finite or sufficiently large (the continuum limit). Before taking this limit, the theory is finite and mathematically well-defined. Therefore, we can apply various non-perturbative techniques. Here, we perform numerical simulations on finite lattices to calculate hadrons.

To accomplish this, the most time-consuming part of the calculation is the inversion of quark propagation kernels. Numerically, this is an inversion of large sparse complex matrices. For the case of Wilson-type lattice quark actions, the typical size of the matrix is $12 V \times 12 \mathrm{~V}$, where $12=3 \times 4$ is the freedom of color and spin, and $V=N^{4}$ is the lattice volume. Because the condition number is inversely proportional to the quark mass, the inversion is numerically more intensive when we decrease the quark mass. Even with the latest supercomputers, it is difficult to simulate the light $u, d$ quarks directly. Therefore, in addition to the continuum extrapolation, we need to extrapolate the results at typically around the $s$ quark mass to the physical $u, d$ quark mass point. This procedure is called "the chiral extrapolation".

In summary, in order to extract a prediction for the real world, we have to perform continuum and chiral extrapolations. To get a precise and reliable result, it is essential to have good control of these extrapolations. This requires a large-scale systematic calculation, and thus huge computer power. The power of vector supercomputers in 1980's was not sufficient, which motivated several groups of lattice physicists to construct parallel computers dedicated to lattice calculations [1].

The historical development of computer speed is shown in Fig. 1. The frontier of speed has been advanced by parallel computers since around 1990. Dedicated machines developed by physicists contributed much to this trend. At the University of Tsukuba, we 


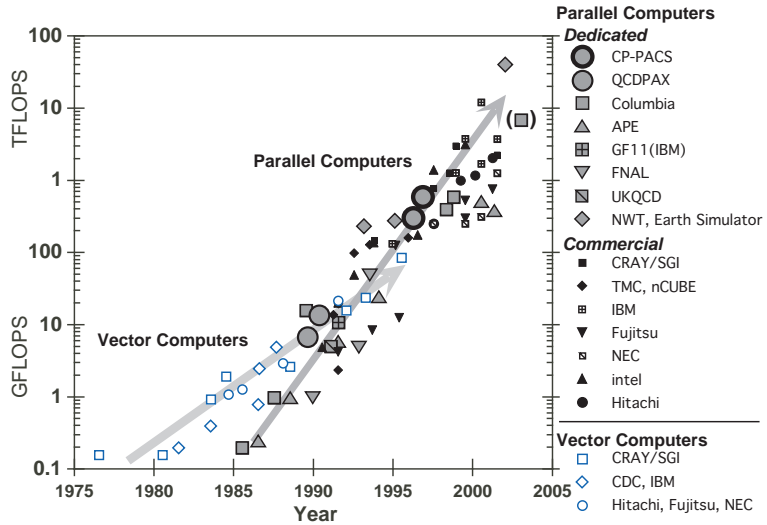

Figure 1: Development of the computer speed.

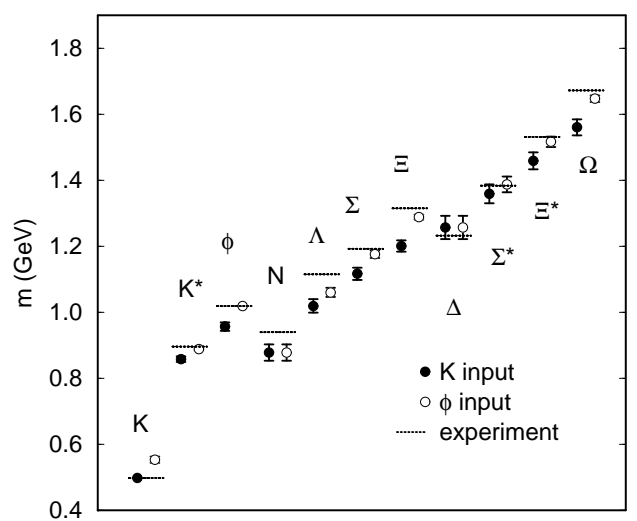

Figure 2: Quenched light hadron spectrum [5].

have developed two machines, QCDPAX (1990) [3] and CP-PACS (1996) [2]. CP-PACS is a parallel computer achieving peak performance of 614.4 GFLOPS with 2048 singleprocessor nodes. Since the first power-on in 1996, intensive lattice QCD calculations have been made on CP-PACS. In the following, I focus on the studies of hadron spectrum and light quark masses.

\section{Light hadron mass spectrum}

Precise calculation of the hadron mass spectrum directly from the first principles of QCD is one of the main goals of lattice QCD. Because the computer power required is enormous, we study the issue step by step as follows:

Step 1 : Calculate in the quenched approximation, in which the effects of dynamical pair creation and annihilation of quarks are neglected.

Step 2 : Include the dynamical $u, d$ quarks in a degenerate approximation, while heavier quarks are treated in the quenched approximation (two-flavor full $Q C D$ ).

Step 3 : Include the dynamical $s$ quark $(2+1$ flavor full $Q C D)$.

Steps $4,5, \cdots$ : Introduce the $u, d$ mass difference, dynamical $c$ quark, etc.

\subsection{Quenched studies}

With the quenched approximation, we can reduce the computer time by a factor of several hundred preserving the basic properties of QCD (confinement, asymptotic freedom, and spontaneous breakdown of chiral symmetry). The effects of the approximation are expected to be about $10 \%$ in the spectrum.

The first studies in this approximation had already been made in early 80's. The issue turned out to be quite tough and computationally demanding. Actually, it took about ten years until the first systematic study, performing all the extrapolations, was attempted in 1993 [ [4]. From this study, the quenched light hadron spectrum was found to be consistent with experiment within the errors of about $10 \%$. However, the quality of data was insufficient to test the accuracy of numerical extrapolations, and also the final errors were too large to resolve the quenching artifact.

The status was significantly improved by CP-PACS [5]. From an intensive computation involving about 100 times more floating-point calculations than the previous studies, it 


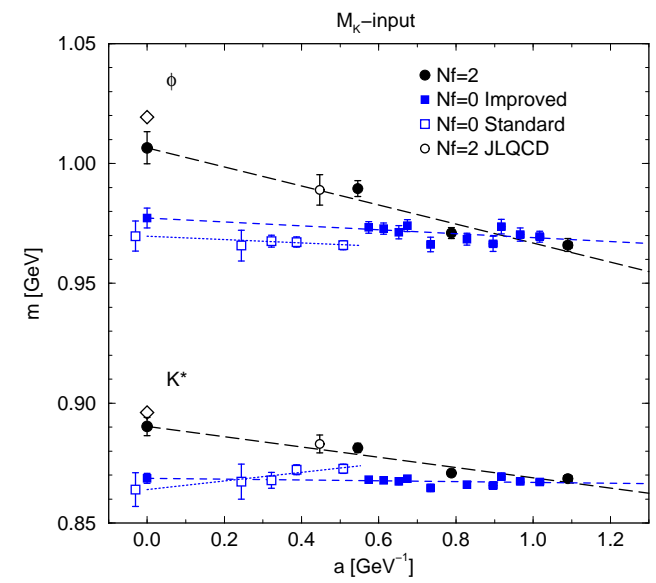

Figure 3: Continuum extrapolation of vector meson masses $M_{\phi}$ and $M_{K^{*}}$ in two-flavor full QCD $\left(N_{f}=\right.$ 2) and quenched QCD $\left(N_{f}=0\right)$, using $M_{K}$ as input [7]. Open circles are recent results using a different improved lattice action [8].

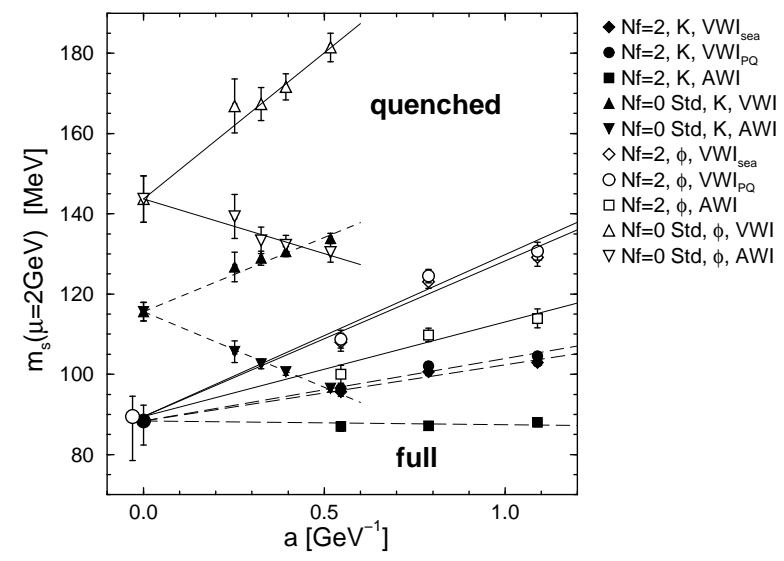

Figure 4: Continuum extrapolation of the $s$ quark mass $m_{s}$ in the $\overline{\mathrm{MS}}$ scheme at $2 \mathrm{GeV}$ from twoflavor [7] and quenched QCD [5]. Quenched masses are shown by triangles. Filled and open symbols are for the $M_{K}$ and $M_{\phi}$ inputs, respectively.

became possible to perform systematic tests on the quality of the extrapolations. Errors in the final results for the light hadron spectrum are now confidently estimated to be less than about 1\% for mesons and about 3\% for baryons. These errors include statistical and all systematic errors except for those from the quenched approximation itself.

The quenched light hadron spectrum is shown in Fig. 2. Experimental values of meson masses $-M_{\pi}, M_{\rho}$, and either $M_{K}$ or $M_{\phi}$ - are used as inputs to fix the lattice spacing $a$ (or the strong coupling constant), the average $u, d$ quark mass $m_{u d}$, and the $s$ quark mass $m_{s}$. Masses for other hadrons are among the predictions of QCD. From Fig. 2, we find that the global pattern of the spectrum is well reproduced. At the same time, we clearly see that the quenched spectrum deviates from experiment by about $10 \%$ (7 standard deviations) for the worst case: Results from the $M_{K^{-}}$-input and $M_{\phi}$-input are discrepant by, and the hyperfine splitting between $K$ and $K^{*}$ mesons is smaller than experiment by about $10 \%$. Decuplet baryon splittings are also small. Because all other systematic errors are well controlled, we identify the discrepancy as quenching artifact.

\subsection{Two-flavor full QCD}

From the quenched simulation we find that, to calculate hadronic quantities with precision better than $10 \%$, we have to incorporate dynamical quarks. A naive extension of the quenched simulation to full QCD is difficult because several hundred times more computer time is required. A partial solution is given by improvement of the lattice theory, whereby continuum properties are realized on coarser lattices. From a preparatory study [6], we find that the combination of renormalization-group improved glue action and clover-improved Wilson quark action is effective in removing major lattice artifacts. This reduces the computer time by a factor of about ten.

Although more might still be hoped for, we can start the first systematic studies of twoflavor full QCD on the CP-PACS performing both chiral and continuum extrapolations [7]. To identify dynamical quark effects clearly, we carried out another quenched simulation using the same improved action.

Figure 3 shows the continuum extrapolation of vector meson masses. We find that the 
Table 1: Light quark masses in $\mathrm{MeV}$ in the $\overline{\mathrm{MS}}$ scheme at $2 \mathrm{GeV}[7]$.

\begin{tabular}{lcccc}
\hline & $m_{u d}$ & $m_{s}(K$-input $)$ & $m_{s}(\phi$-input $)$ & $m_{s} / m_{u d}$ \\
\hline$N_{f}=0$ Stand. & $4.57 \pm 0.18$ & $116 \pm 3$ & $144 \pm 6$ & $\approx 25-31$ \\
$N_{f}=0$ Impr. & $4.36_{-0.17}^{+0.14}$ & $110_{-4}^{+3}$ & $132_{-6}^{+4}$ & $\approx 25-30$ \\
$N_{f}=2$ & $3.44_{-0.22}^{+0.14}$ & $88_{-6}^{+4}$ & $90_{-11}^{+5}$ & $26 \pm 2$ \\
\hline
\end{tabular}

two quenched results (open and filled squares) lead to universal values in the continuum limit $a=0$. They deviate from the experimental values (diamonds) as noted in the previous subsection. On the other hand, the full QCD results (circles) extrapolate to values much closer to experiment. Accordingly, we find no big discrepancies between the results from $M_{K}$ and $M_{\phi}$ inputs. This means that the quenching artifacts in the spectrum are mostly removed by introducing dynamical $u, d$ quarks.

The whole light hadron spectrum can now be approximately reproduced from QCD by adjusting just three parameters; the strong coupling constant, $m_{u d}$ and $m_{s}$. This provides us with a strong confirmation that QCD is correct also at low energies. Remaining small deviations from experiment may be explained by the quenched approximation of the $s$ quark. In order to confirm this, however, uncertainties from the chiral and continuum extrapolations should be reduced to the level of our quenched study. This goal is reserved for future work.

\section{Light quark masses}

Adjustment of $m_{u d}$ and $m_{s}$ in the calculation of the hadron spectrum provides us with the most direct determination of quark masses from QCD. Fig. 4 shows $m_{s}$ in the $\overline{\mathrm{MS}}$ scheme at $\mu=2 \mathrm{GeV}$ as functions of the lattice spacing $a$. Results from the quenched improved action, which are consistent with the quenched standard action in the continuum limit, are omitted for clarity.

On the lattice, there are several alternative definitions for the quark mass, with the difference being $O(a)$. In Fig. 14, they are denoted as VWI and AWI (vector and axialvector Ward-Takahashi identity). See [5, 7] for details. As shown in Fig. 1, different definitions lead to different values of $m_{q}$ at finite lattice spacings. This has been a big source of uncertainty in the previous calculations. From Fig. \&, we see that they converge to a universal value in the continuum limit, as theoretically expected. This confirms the quality of our calculations.

The quenched value for $m_{s}$, however, differs by about $20 \%$ between $M_{K}$-input and $M_{\phi}$-input. This is equivalent to the quenched artifact discussed in Sec. 3.1. We note that the quenched artifact is larger in the light quark masses than in the hadron spectrum. When we turn on the dynamical $u, d$ quarks, the discrepancy between the inputs disappears within our errors, in accord with the fact that the full QCD reproduces the hadron spectrum better (Sec. 3.2).

The light quark masses in the continuum limit are summarized in Table 1. The errors include our estimates for systematic errors from chiral and continuum extrapolations and renormalization factors. We note that the masses from two-flavor QCD are 20-30\% smaller than those from the quenched QCD. In particular, our $s$ quark mass in $N_{f}=2$ QCD is about $90 \mathrm{MeV}$, which is significantly smaller than the value $\approx 150 \mathrm{MeV}$ often used in phenomenology. Our results are, however, consistent with recent estimates $m_{s}=83-130$ $\mathrm{MeV}$ and $m_{u d}=3.4-5.3 \mathrm{MeV}$ from QCD sum rules [9] and $m_{s} / m_{u d}=24.4 \pm 1.5$ from 
one-loop chiral perturbation theory [10].

\section{Conclusions}

An intensive calculation of the light hadron spectrum in the quenched approximation has revealed discrepancies of about $10 \%$ from experiment. With two flavors of dynamical $u, d$ quarks, the first systematic study performing both the continuum and chiral extrapolations has shown that the discrepancies are mostly removed, providing us with a strong confirmation that QCD is the correct theory of quarks at low energies as well.

Precision calculation of hadron masses enables us to determine fundamental parameters of quarks directly from the first principles of QCD. We find that the dynamical quark effect is as large as 20-30\% in light quark masses. Noticeable and sizable dynamical quark effects are observed also in B meson decay constants [11], the equation of state at high temperatures [12], and the topological structure of the QCD vacuum [13]. At the same time, new types of lattice fermions have begun to be applied to study hadronic matrix elements relevant to the $\Delta I=1 / 2$ rule and the CP violation parameters [14].

Because a shift in fundamental parameters can have significant implications to phenomenological studies of the standard model, it is urgent to evaluate dynamical quark effects in other hadronic quantities as well. The influence of dynamical $s$ quark should also be studied. Studies in these directions are under way to clarify the precise structure of the standard model.

The studies presented in this report have been performed by the CP-PACS Collaboration. I thank the members of the Collaboration for discussions and comments. This work is in part supported by the Grants-in-Aid of Ministry of Education, Science and Culture (Nos. 12304011 and 13640260) and JSPS Research for Future Program.

\section{References}

[1] For recent reports, see Proc. Lattice 2000, Nucl. Phys. B (Proc. Suppl.) 94 (2001).

[2] Y. Iwasaki, Nucl. Phys. B (Proc. Suppl.) 60A (1998) 246, For further details, see http://www.rccp.tsukuba.ac.jp.

[3] Y. Iwasaki et al., Comp. Phys. Commun. 49 (1988) 363.

[4] F. Butler et al., Nucl. Phys. B430 (1994) 179.

[5] S. Aoki et al. (CP-PACS Collab.), Phys. Rev. Lett. 84 (2000) 238.

[6] S. Aoki et al. (CP-PACS Collab.), Phys. Rev. D60 (1999) 114508.

[7] A. Ali Khan et al. (CP-PACS Collab.), Phys. Rev. Lett. 85 (2000) 4674; heplat/0105015.

[8] S. Aoki et al. (JLQCD Collab.), hep-lat/0110179; T. Kaneko, hep-lat/0111005.

[9] R. Gupta and K. Maltman, DPF2000 (2001) hep-ph/0101132.

[10] H. Leutwyler, Phys. Lett. B378 (1996) 313.

[11] A. Ali Khan et al. (CP-PACS Collab.), Phys. Rev. D64 (2001) 034505; ibid. D64 (2001) 054504. 
[12] M. Okamoto et al. (CP-PACS Collab.), Phys. Rev. D60 (1999) 0094510; A. Ali Khan et al. (CP-PACS Collab.), ibid. D63 (2001) 034502; ibid. D64 (2001) 074510; Y. Namekawa et al. (CP-PACS Collab.), ibid. D64 (2001) 074507.

[13] A. Ali Khan et al. (CP-PACS Collab.), Phys. Rev. D64 (2001) 114501.

[14] A. Ali Khan et al. (CP-PACS Collab.), Phys. Rev. D63 (2001) 114504; ibid. D64 (2001) 114506; J-I. Noaki et al. (CP-PACS Collab.), hep-lat/0108013 